“ (C) 2013 IEEE. Personal use of this material is permitted. Permission from IEEE must be obtained for all other uses, in any current or future media, including

reprinting/republishing this material for advertising or promotional purposes, creating new collective works, for resale or redistribution to servers or lists, or reuse of any copyrighted component of this work in other works." 


\title{
Development of an assistive patient mobile system for hospital environments
}

\author{
Huy Hoang Nguyen, Tuan Nghia Nguyen, Raymont Clout, Alexander Gibson \\ and Hung T. Nguyen, Senior Member, IEEE
}

\begin{abstract}
This paper presents an assistive patient mobile system for hospital environments, which focuses on transferring the patient without nursing help. The system is a combination of an advanced hospital bed and an autonomous navigating robot. This intelligent bed can track the robot and routinely navigates and communicates with the bed. The work centralizes in building a structure, hardware design and robot detection and tracking algorithms by using laser range finder. The assistive patient mobile system has been tested and the real experiments are shown with a high performance of reliability and practicality. The accuracy of the method proposed in this paper is $91 \%$ for the targeted testing object with the error rate of classification by $6 \%$. Additionally, a comparison between our method and a related one is also described including the comparison of results.
\end{abstract}

\section{INTRODUCTION}

The hospital bed plays an important role for the patient during their entire stay in hospital. It not only helps the patient to be comfortable while receiving medical treatment but also the best must be mobile within hospital environment. In recent years, the emergence of advanced technologies makes the hospital bed more flexible and extra functions have been added to assist patient movements. This is called an intelligent bed. Improving the hospital bed, therefore decreasing the physical demands of nursing work, is the main target which researchers have been focusing on.

One aspect of this research is the design of a multifunctional bed which assists bedridden patients to change their positions without nursing help [1]. Normally, to adjust the bed posture, the nurse does it by herself. By using an intelligent bed the patient has control without the need for training and assistance, [2] which gives a solution to this problem. However, both systems still require nursing help if the patient is to be moved from one room to another. Our research approach focuses on decreasing this nursing support by developing a smart bed, specifically for transporting the patient within the hospital environment.

With this approach, our design is called an assistive patient mobile system (APMS). This system is a combination of a hospital bed and an autonomous robot named Turtlebot. The Turtlebot automatically navigates in the clinical environment and works as a guide for the intelligent bed which follows the robot. Based on our related

Hoang Huy Nguyen, Tuan Nghia Nguyen, Raymont Clout, Alexander Gibson and Hung T. Nguyen are with the Centre for Health Technologies, Faculty of Engineering and Information Technology, University of Technology, Sydney, Broadway, NSW 2007, Australia, (e-mail: Huy.H.Nguyen-2@student.uts.edu.au; Tuannghia.nguyen@uts.edu.au; Ray.Clout@uts.edu.au; Alexander.Gibson@uts.edu.au Hung.Nguyen@uts.edu.au ) work on the intelligent wheel chair [3], the system structure of the hospital bed is designed to operate as a semiautonomous bed.

To achieve this goal, object detection methods need to be considered and applied. There are many different types of objects in the hospital environment such as: humans, wheelchairs and various obstacles including the Turtlebot. Thus, distinguishing the types of objects is essential to let the intelligent bed follow the target. Moreover, object detection also affects the obstacle avoidance process which is necessary to any mobile system.

Various approaches to object detection in robot following target algorithms have been proposed such as using voice recognition sensors, infrared cameras, and CCD cameras. In [4], face detection is applied for tracking a person based on camera vision which generates a robust measurement of the person's distance from the robot by considering 16 sonar scans and tactile sensor readings. Another approach is a combination of a sonar ring of sensors, laser range sensor and vision camera which increases the feasibility of the robot to follow a human in a smoky environment [5]. Two methods in [4], [5] also have their own advantages, however, their systems are expensive and it would be a substantial increase in cost if one of these methods were applied to our system. With the purpose of robot detection, using only a laser range finder is enough for our approach.

There are several object detection methods based exclusively on the laser range finder. Recently, a study [6] suggested a method based on three features of the object using Support Vector Data Description (SVDD) to construct the boundary of the data set regarding to its features. Using this method, it is time consuming to build the boundary for the data set. The larger the data set, the longer the SVDD method takes to process. Moreover, its shape contour is sensitive to generate. Instead of using SVDD, we applied a Gaussian Distribution Method (GDM) to build a new robot detection algorithm for this study.

In this paper, we propose a new assistive patient mobile system for a hospital context. A modified robot detection algorithm is applied to control the intelligent bed in order to follow a guide robot in the hospital environment. The paper is organized as follows. In section II, the APMS structure will be discussed and advanced robot detection method will be introduced in section III. Experimental results will be shown in Section IV. The last section will illustrate our conclusion. 


\section{SYSTEM DESIGN AND DETECTION METHOD}

\section{A. System design}

There are two main parts in the assistive patient mobile system. The first one is a semi-autonomous mobile system with the hardware overview and the second one is an autonomous robot named Turtlebot shown in Fig.1.

The hospital bed: the central processor system is the combination between an ARM 32bit embedded system and a $\mathrm{PC}$, which is Mac Mini. RS232 and USB connection are used to connect both of them. With the purpose of allowing the patient to interact with the system, our structure has been designed to receive the commands from the user through iPad, Joystick and button pad. In addition to the wheel encoders to measure the position of the mobile device, a laser range finder URG-04LX is used to measure the distance of background and foreground subjects. The URG04LX which can provide accurate range data, high resolution, and high speed streaming data will be used in this study. There is an open board named Phiget 888 for obtaining parameters from a set of optional sensors. A quad KTA198 board and HDC2450 controller are used to control the low-level components of the mobile device.

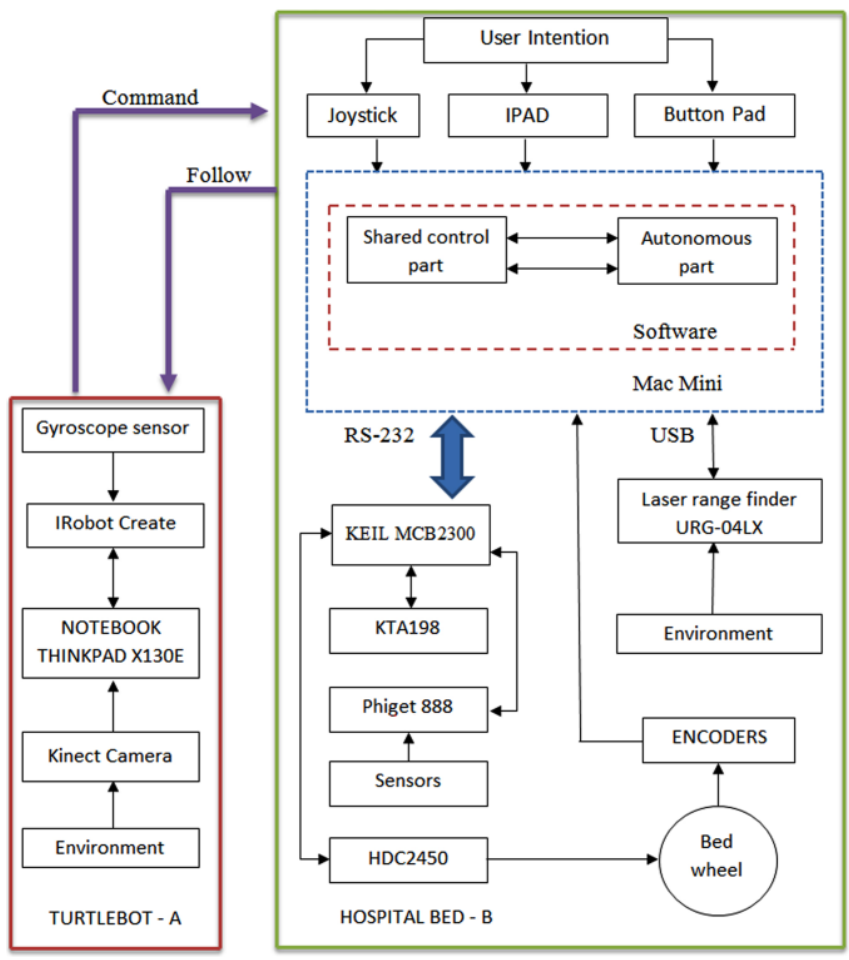

Fig.1.The system structure

The hospital bed is designed based on achievement of our previous system: the intelligent wheelchair [3]. It is a semiautonomous system. In general, a semi-autonomous system consists of two main parts: shared control part and autonomous part. Different from a fully-autonomous system, shared control part of a semi-autonomous system allows users to communicate with the system through commands from users and autonomous part to reach decisions. With this type of system, the hospital bed can receive commands from users through various kinds of interfaces (i.e. ipad, joystick, button pad) and now from Turtlebot by assembled programming. The program of APMS is built on Labwindow/CVI 2010.
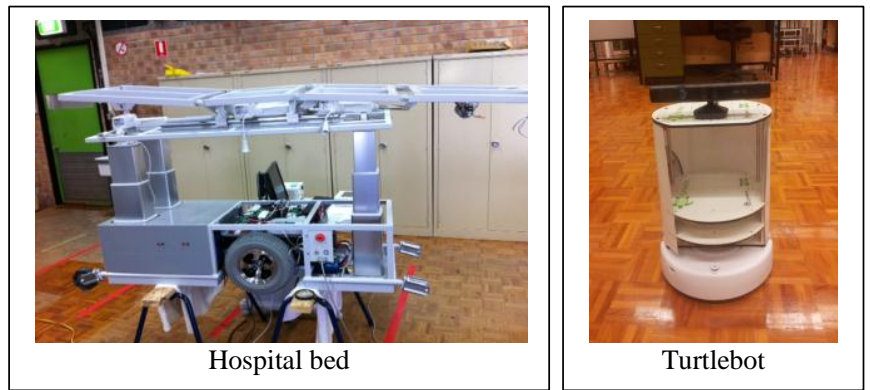

Fig.2.The hardware system

The Turtlebot: works as a guidance robot, and can autonomously navigate with SLAM algorithm. This robot is integrated with an IRobot Create, a notebook with $1.3 \mathrm{GHz}$ Dual Core Processor and Kinect camera. The Turtlebot transmits and receives information with the mobile device robot through $\mathrm{WiFi}$.

Our hardware system is shown in Fig. 2: the hospital bed, the laser range finder URG-04LX installed on the bed and the Turtlebot.

\section{B. Detection method}

As the Turtlebot has the shape of a cylinder with the constant parameters of radius $(160 \mathrm{~mm})$ and height $(400$ $\mathrm{mm}$ ), circle detection is applied for Turtlebot detection.

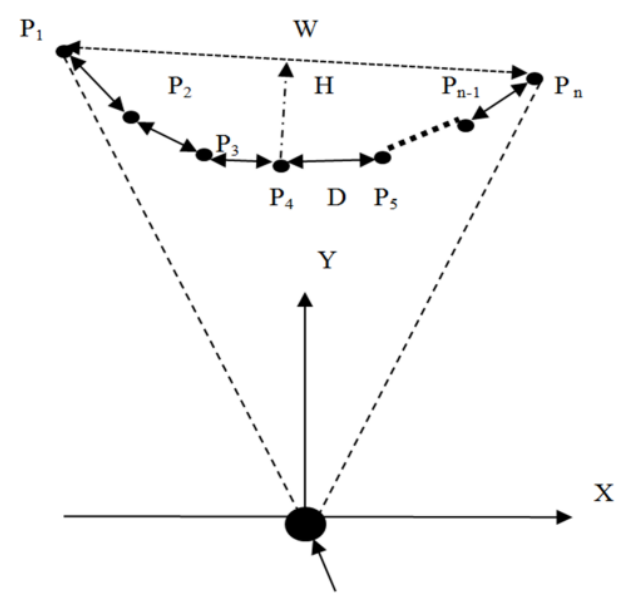

Laser Range Finder

Fig.3. Scan points and features of the Turtlebot

URG-04LX Laser range finder (LRF) is used for this application. The resolution scan angle of LRF is $1.08^{\circ}$ which means there are 171 points received from LRF every scan. Data from laser range finder is the set of points and distance between these points and LRF. As LRF scan on one plane, one part of the circle is detected from LRF. Therefore, to detect the circle we identify the arc in Turtlebot which is seen by LRF. Basing on [6] we define four features of the Turtlebot including: W, D, H, G (Fig.3). According to [6], four features are defined below: 
- $\mathrm{D}$ is defined as the distance between two nearest points:

$$
D=\overline{P_{l} P_{l+1}}
$$

- $\mathrm{G}$ is the girth of the Turtlebot which is defined by the following equation:

$$
G=\sum_{i=1}^{n} \overline{P_{l} P_{l+1}}
$$

- $\mathrm{W}$ is the distance between the start and end point of the cluster point of the Turtlebot and defined by the following equation:

$$
W=\overline{P_{1} P_{n}}
$$

- $\mathrm{H}$ is the biggest distance between one point of the point cluster to the line of the start and end point of this:

$$
H=\max \left(\frac{\left|a \cdot x_{P_{i}}+b \cdot y_{P_{i}}+c\right|}{\sqrt{a^{2}+b^{2}}}\right)
$$

Where $a, b, c$ are parameters of the line equation through the start and end points of the point cluster of the Turtlebot.

In this study, we aim to investigate the correlation between three features G, W, H of the Turtlebot. The objects are divided as Turtlebot or non-Turtlebot. We will classify the data belonging to Turtlebot or non-Turtlebot objects. The target class is the Turtlebot and the outlier is the nonTurtlebot. One of the popular, simple and effective classification methods is the Gaussian distribution method [7]. For our study, we assume that the density estimate avoided and just applied the Mahalanobis distance in our problem [8]:

$$
F(x)=(x-\mu)^{T} \Sigma^{-1}(x-\mu)
$$

The classifier is defined as:

$$
h(x)=\left\{\begin{array}{lll}
\text { target } & \text { if } & F(x) \leq \theta \\
\text { outlier } & \text { if } & F(x)>\theta
\end{array}\right.
$$

The mean $\mu$ and the covariance matrix $\sum$ are sample estimation of data $x$. The threshold $\theta$ is set according to the target error.

We set up two normal data $x_{i}^{W_{i} G_{i}}$ and $x_{j}^{G_{j} H_{j}}$ of features $\left(W_{i}, G_{i}\right)$ and $\left(G_{j}, H_{j}\right)$, respectively. Basing on the Gaussian distribution, we build two boundaries for two data, $B_{W G}, B_{G H}$.

\section{Robot detection algorithm:}

The robot detection algorithm combines three steps in which step 3 includes a for loop where conditions if-then must be obeyed in order.

- Step 1 - LRF data acquisition and calculation: Calculating the position of points in coordinate $(\mathrm{X}, \mathrm{Y})$, the centre of the coordinate is the position of LRF.

- Step 2 - Pre-processing: foreground data will be kept after using a background subtraction algorithm [9] and then is clustered by feature D. After that, $\mathrm{N}$ clusters $(\mathrm{C})$ are created from foreground data. $\mathrm{N}$ is the number of clusters which is collected after clustering foreground data. $W_{k}, G_{k}$ and $H_{k}$ are features of cluster $C_{k}$ with $k \in N$.

- Step 3 - FOR $k=1$ TO N

$$
\begin{gathered}
\text { IF }\left(W_{k}, G_{k}\right) \in B_{W G} \text { THEN } \\
\text { IF }\left(G_{k}, H_{k}\right) \in B_{G H} \text { THEN } \\
C_{k}=\text { TURTLEBOT } \\
\text { ENDIF } \\
\text { ENDIF } \\
\text { ENDFOR }
\end{gathered}
$$

\section{RESULTS}

For initial setting up, the distance between the laser range finder and the Turtlebot is $1000(\mathrm{~mm})$ with the variable range of $10 \%$. We conducted the robot detection algorithm in an experimental environment with two other objects, a human leg and a trash can which also are popular in indoor environments. Assuming that, the trash can has the radius of $140(\mathrm{~mm})$ and height of $400(\mathrm{~mm})$ and human leg is of a male wearing a long trouser. All experiment results are calculated basing on 750 samples with the distance of $1000(\mathrm{~mm}) \pm$ $10 \%$ from the laser range finder. The distribution of 3 features W, G, H is presented in Fig.4

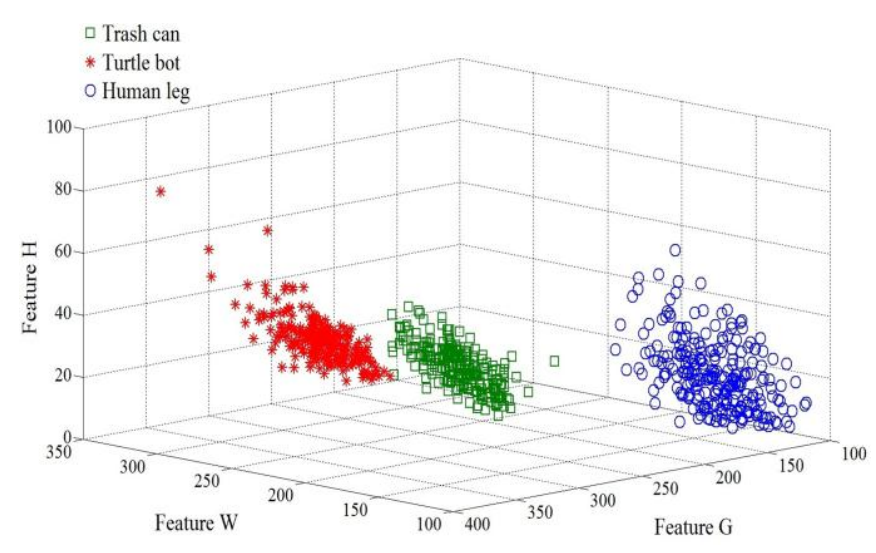

Fig.4. Distribution of 3 features

Applying Gaussian distribution method for 200 Turtlebot samples $\left(x_{i}^{W_{i} G_{i}}\right)$. The Mahalanobis distance is given by [6]:

$$
\begin{gathered}
F\left(\begin{array}{c}
W_{i} \\
G_{i}
\end{array}\right)=\left(d_{G_{i}}, d_{W_{i}}\right)\left(\begin{array}{cc}
\sigma_{G G} & \sigma_{G W} \\
\sigma_{W G} & \sigma_{W W}
\end{array}\right)\left(d_{G_{i}}, d_{W_{i}}\right)^{T} \\
d_{G_{i}}=G_{i}-G_{\text {mean }} \text { and } d_{W_{i}}=W_{i}-W_{\text {mean }} \\
G_{\text {mean }}=\frac{\sum_{i=1}^{200} G_{i}}{200} \text { and } W_{\text {mean }}=\frac{\sum_{i=1}^{200} W_{i}}{200}
\end{gathered}
$$

Changing the threshold $\theta$ from 0.2 to 0.05 with step of 0.05 , we got 4 contours $(1,2,3,4)$ respectively. We use 100 samples of Turtlebot data to test the accuracy of algorithm. The result of this test is shown in Fig.5. The contour (4) achieved the highest percentage of $95 \%$ and the contour (1) obtained the lowest of $63 \%$. Similarly work of the boundary $B_{W G}$, the results of the boundary $B_{G H}$ are $98 \%$ and $73 \%$ for contour 4 and 1, respectively. Again, we test with 100 samples of non-Turtlebot and the results of testing the false positive rate of this method are described in Table I where the false positive rate is the proportion of the number of non-Turtlebot testing data inside the boundary and the total number of non-Turtlebot testing data. 


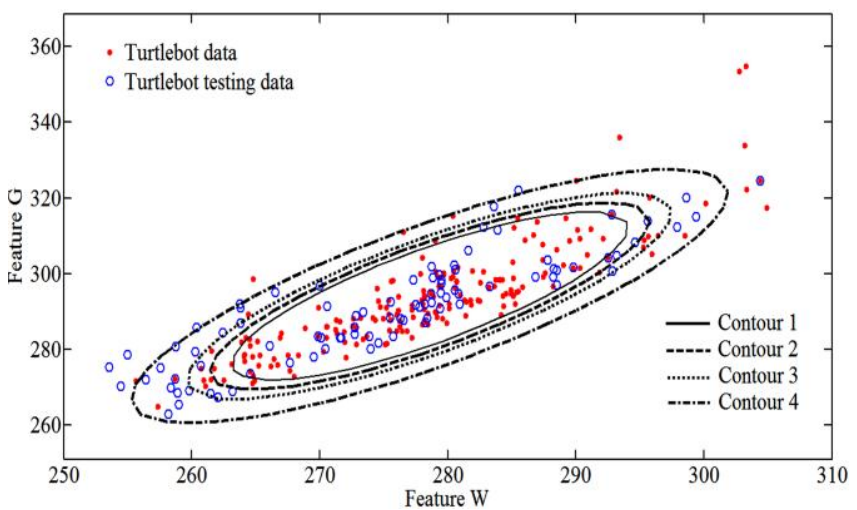

Fig.5.The Boundary $B_{W H}$ with $\theta=0.2,0.15,0.1,0.05$

TABLE I. THE FALSE POSITIVE RATE OF NON-TURTLEBOT DETECTION

\begin{tabular}{|c|c|c|c|c|}
\hline & Contour 4 & Contour 3 & Contour 2 & Contour 1 \\
\hline$B_{W G}$ & $1 \%$ & $0 \%$ & $0 \%$ & $0 \%$ \\
\hline$B_{G H}$ & $3 \%$ & $2 \%$ & $2 \%$ & $2 \%$ \\
\hline
\end{tabular}

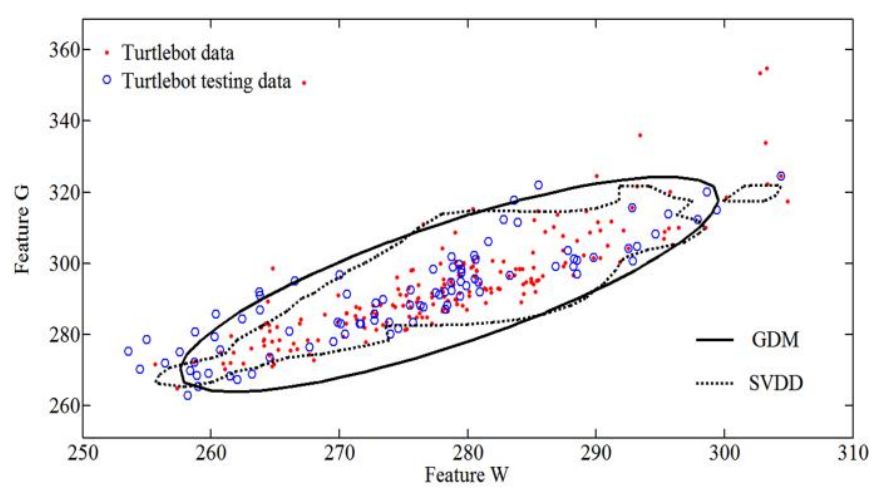

Fig.6. A comparison between SVDD and GDM at error rate of $16 \%$

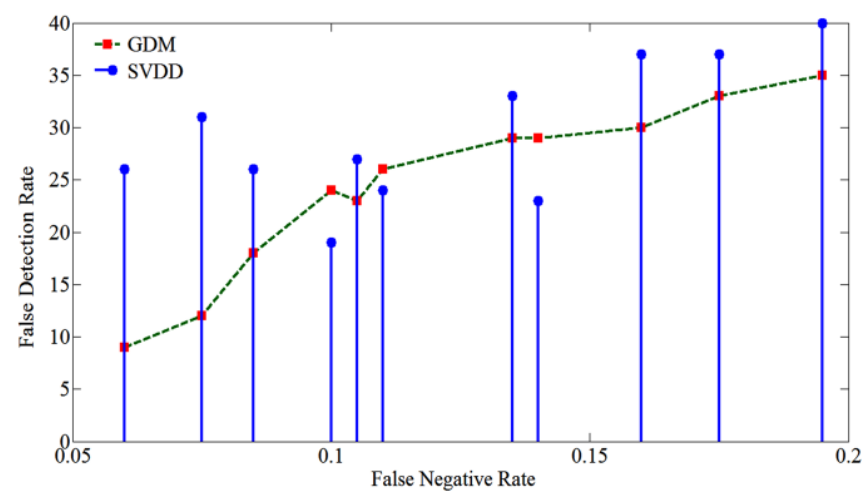

Fig.7. Correlation between false negative and false detection rate of classifiers

For comparison, we ran two methods in parallel, GDM and SVDD at the same false negative rate of $16 \%$ (as shown in Fig. 6). The false negative rate here is the proportion of number of target data outside the boundary and total number of target data. The graph indicates that the boundary generated by GDM is larger than that generated by SVDD, which means it has a higher ability to cover more data.

Figure 7 shows the correlation between a false negative rate and a false detection rate of classifier, where the false detection rate is the proportion of number of Turtlebot testing data outside the generated boundary and total number of Turtlebot testing data. The result demonstrates that GDM gives a lower false detection rate compared to SVDD. The false negative rate of GDM is increased gradually regarding the increase of the false negative rate, while SVDD releases a high and unstable false detection rate depending on the training process of mapping. GDM allows adjusting false negative rate flexibly and can achieve higher accuracy and is more reliable than SVDD. It can be seen from Fig.7 that by GDM, a false negative rate at $6 \%$ got the lowest false detection rate of $9 \%$ which means the accuracy of our study in Turtlebot detection is $91 \%$. We concluded that the best threshold of false negative rate $\theta$ for our method is $6 \%$.

\section{CONCLUSION}

We have designed a new system by combining the hospital bed and the Turtlebot with the purpose of assisting patients when moving in a hospital environment. An improved robot detection algorithm has also been developed in this paper. Experimental results show that the proposal algorithm can detect 3 different objects with accuracy rate of $91 \%$. Our feature research will focus on developing an advanced real-time control strategy for the hospital bed to guarantee the stability of the overall system and perform intelligent tasks such as object following, obstacle avoidance and sharing control in various environments.

\section{REFERENCE}

[1] P. Shih-Wei, L. Feng-Li, and F. Li-Chen, "Mechanism Design and Mechatronic Control of a Multifunctional Test Bed for Bedridden Healthcare," Mechatronics, IEEE/ASME Transactions on, vol. 15, pp. 234-241, 2010.

[2] K. Shyu, Y. J. Chiu, P. L. Lee, M. H. Lee, J. J. Sie, C. H. Wu, Y. T. $\mathrm{Wu}$, and P. C. Tung, "Total Design of an FPGA-Based Brain Computer Interface Control Hospital Bed Nursing System," Industrial Electronics, IEEE Transactions on, vol. PP, pp. 1-1, 2012.

[3] A. V. Nguyen, S. Su, and H. T. Nguyen, "Development of a Bayesian recursive algorithm to find free-spaces for an intelligent wheelchair," in Engineering in Medicine and Biology Society,EMBC, 2011 Annual International Conference of the IEEE, 2011, pp. 7250-7253.

[4] P. Vadakkepat, P. Lim, L. C. De Silva, J. Liu, and L. Li Li, "Multimodal Approach to Human-Face Detection and Tracking," Industrial Electronics, IEEE Transactions on, vol. 55, pp. 1385-1393, 2008.

[5] J. Sales, R. Marín, E. Cervera, S. Rodríguez, and J. Pérez, "MultiSensor Person Following in Low-Visibility Scenarios," Sensors, vol. 10, pp. 10953-10966, 2010.

[6] C. Woojin, K. Hoyeon, Y. Yoonkyu, M. Chang-Bae, and P. Jooyoung, "The Detection and Following of Human Legs Through Inductive Approaches for a Mobile Robot With a Single Laser Range Finder," Industrial Electronics, IEEE Transactions on, vol. 59, pp. 3156-3166, 2012.

[7] C. M. Bishop and G. Hinton, Neural Networks for Pattern Recognition: Clarendon Press, 1995.

[8] D. M. J. Tax. (2012). DDtools, the Data Description Toolbox for Matlab.

[9] H. Xiangqi, T. Sasaki, H. Hashimoto, and F. Inoue, "Circle detection and fitting based positioning system using laser range finder," in System Integration (SII), 2010 IEEE/SICE International Symposium on, 2010, pp. 442-447. 